\title{
Superconducting Quantum Interference Device
}

National Cancer Institute

\section{Source}

National Cancer Institute. Superconducting Quantum Interference Device. NCI

Thesaurus. Code C124066.

A device designed to measure extremely weak magnetic fields. It is comprised of a Josephson junction with two superconducting loops separated by an insulating layer thin enough to allow electrons to pass through. 